\title{
Gastroesophageal Reflux Disease in Patients with Eustachian Tube Catarrh
}

\author{
${ }^{1}$ Anuja Bhargava, ${ }^{2}$ Meenu Cherian, ${ }^{3}$ Tambi A Cherian, ${ }^{4}$ Sanjay Gupta
}

\section{ABSTRACT}

Background: Eustachian tube catarrh could be due to laryngopharyngeal reflux besides other causes.

Objectives: To assess gastroesophageal reflux disease (GERD) in patients with Eustachian tube catarrh and the effect of proton pump inhibitors on symptoms of Eustachian tube disease.

Methodology: A total of 50 patients were selected with symptoms of Eustachian tube catarrh and evaluated prospectively in the ENT Outpatient Department of the Pondicherry Institute of Medical Sciences, Puducherry.

Results: The group consisted of $15(30 \%)$ males and $35(70 \%)$ females. The largest group was of the age of 45 years and above (44\%). The most common symptom of Eustachian tube catarrh was itching ( $84 \%$ ), followed by otalgia ( $76 \%$ ) and popping sensation on swallowing (74\%). On otoscopic examination, the commonest grade of tympanic membrane retraction was grade I $(57 \%)$, on tympanometry $90 \%$ of cases had middle ear pressure in range -100 to +100 . The middle compliance ranged from 0.5 to 1.75 (normal) in $86 \%$ of the cases. The tympanomeric curve was type $A$ (normal) in $78 \%$ of the cases and type $C$ in $8 \%$ of the cases. At the end of 4 and 8 weeks, the response of treatment to proton pump inhibitors was significantly higher $(z=3.53, p<0.05)$ in the studied group.

Conclusion: Laryngopharyngeal reflux (LPR) could be an important etiological factor in Eustachian tube catarrh. The treatment, with proton pump inhibitors, of Eustachian tube catarrh with no local identifiable cause, could be very useful to this subsect of patients.

Keywords: Eustachian tube catarrh, Gastroesophageal reflux, Laryngopharyngeal reflux, Proton pump inhibitors.

How to cite this article: Bhargava A, Cherian M, Cherian TA, Gupta S. Gastroesophageal Reflux Disease in Patients with Eustachian Tube Catarrh. Int J Phonosurg Laryngol 2015;5(2): 61-66.

\footnotetext{
${ }^{1}$ Assistant Professor, ${ }^{2-4}$ Professor

${ }^{1}$ Department of ENT and Head and Neck Surgery, Era Lucknow Medical College and Hospital, Lucknow, Uttar Pradesh, India

2,3 Department of ENT and Head and Neck Surgery, Pondicherry Institute of Medical Sciences, Puducherry, Tamil Nadu, India

${ }^{4}$ Department of Community Medicine, People's College of Medical Sciences and Research, Bhopal, Madhya Pradesh, India

Corresponding Author: Anuja Bhargava, Assistant Professor Department of ENT and Head and Neck Surgery, No. 8, Blunt Square, Durgapuri, Lucknow-226004, Uttar Pradesh, India Phone: 91-07897662220, e-mail: anujabhargava@rediffmail. com
}

\section{Source of support: Nil}

Conflict of interest: None

\section{INTRODUCTION}

Eustachian tube patency and its proper functioning are highly essential for the normal maintenance of middle ear function. Obstruction of the Eustachian tube will result in negative pressure in the tympanum and lead to retraction, effusion and other complications.

Eustachian tube has at least three important functions with respect to the middle ear: Ventilation or pressure regulation of the middle ear, clearance or drainage of middle ear secretions into the nasopharynx and protection from nasopharyngeal secretions and sound pressure. The ventilator function is important since a malfunctioning Eustachian tube hampers the function of tympanum and leads to middle ear effusion.

Malfunctioning of eustachian tube opening could be due to laryngopharyngeal reflux (LPR) besides other causes. Previous studies have established that LPR may cause inflammation of the nasopharyngeal end of Eustachian tube and result in Eustachian tube catarrh. This study evaluates gastroesophageal reflux disease (GERD) in patients with Eustachian tube catarrh.

\section{MATERIALS AND METHODS}

A hospital-based prospective study was carried out to assess GERD in patients with Eustachian tube catarrh and to know the effect of proton pump inhibitors on symptoms of Eustachian tube disease in the tertiary care hospital.

A total of 50 patients were selected with purposive sampling and informed consent was obtained from each participant before including in the study, symptoms of Eustachian tube catarrh and effect of proton pump inhibitor evaluated prospectively in the period of 18 months from May 2005 to September 2006 in the ENT Outpatient Department of the Pondicherry Institute of Medical Sciences, Puducherry.

\section{Inclusion Criteria}

Adult patients (more than 18 years of age) with two or more symptoms of tubal occlusion, such as otalgia, hearing loss, itching of the ears, popping sensation on swallowing, tinnitus, giddiness, and willing to participate. 


\section{Exclusion Criteria}

\section{Patient with Tympanic Membrane Perforation}

Eustachian tube dysfunction due to local cause, such as upper respiratory tract infection and allergy, and not willing to participate.

A detailed history, based on a predesigned and pretested questionnaire, was taken from the patients with particular attention to aural and GERD symptoms. If two or more symptoms (such as otalgia, hearing loss, itching of the ear, popping sensation on swallowing, tinnitus and giddiness) of Eustachian tube catarrh were present, the case was symptomatically diagnosed as Eustachian tube catarrh. Gastroesophageal reflux disease was established by any two symptoms, such as heartburn, water brash and belching. Informed consent was taken from all the patients.

Patients underwent ENT examination, impedance audiometry and Eustachian tube function test. None of the patients were subjected to upper gastrointestinal endoscopy as they were not willing for this procedure. All otoscopic examination was done using a Welch Allyn Pneumatic Otoscope. When necessary, cerumen was removed from the external auditory canal with a blunt Jobson-Horne probe or syringing with water at body temperature.

The tympanic membrane was examined otoscopically for presence of the following features: (1) Distortion of cone of light; (2) position of tympanic membrane and (3) decreased mobility. Laryngeal examination was performed by indirect laryngoscopy. Laryngeal changes with erythema of the posterior vocal folds and arytenoids area were termed as posterior laryngitis. Impedance audiometry was done using impedance audiometer (AudioTraveller AA222) (interacoustics) which combines a middle ear analyzer and clinical audiometer. Patients were seated and the hand pencil type probe was inserted in each ear. The following indices were measured-Static compliance, middle ear pressure, tympanogram shapes (Jerger). Following this, patients were subjected to Eustachian tube function test, used in patients with non-perforated drums. It is also known as the Williams' test (Fig. 1). With the AA222, the test is semiautomatic and it suggests the operator how to operate and how to instruct the patient. Three tympanometric curves appear on the screen, which are produced at three different pressures: at baseline, on swallowing (Toynbee test) and on performing the Valsalva.

The three different pressures are also displayed. If the patient has a Eustachian tube dysfunction (ETD) the three pressures will vary only within +10 to $-10 \mathrm{~mm} \mathrm{H}_{2} \mathrm{O}$ of the value and this is labeled as Williams' test negative.

The patients were examined by diagnostic nasal

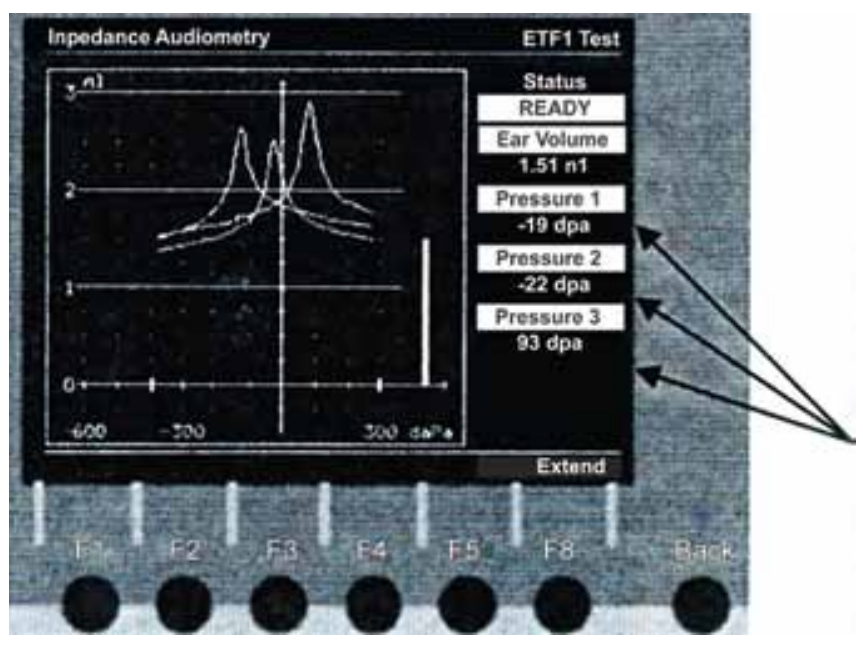

Fig. 1: Eustachian tube function test. Change in peak pressure indicates functioning Eustachian tube

endoscopy under topical anesthesia to look for any local pathology in the nose or nasopharynx.

All patients of Eustachian tube catarrh with or without symptoms of GERD and with no other local symptoms, were treated.

Patients were followed up at an interval of 4 weeks to a maximum of 3 months with a repeat Eustachian tube test and were questioned regarding the spectrum of their symptoms. Statistical analysis was done using appropriate stastical method with the help of expert-like percentage, Z-test and so on.

\section{RESULTS}

The group consisted of $15(30 \%)$ males and $35(70 \%)$ females (Table 1). The majority were in the age groups of 45 years and above ( $44 \%$ ) (Table 2). The most common symptom of Eustachian tube catarrh was itching (84\%) followed by otalgia $(76 \%)$ and popping sensation of swallowing (74\%) (Graph 1). On otoscopic examination, the commonest grade of tympanic membrane retraction was grade I (57\%) on tympanometry (Table 3$), 90 \%$ of cases had middle ear pressure in range -100 to +100 (Table 4). The middle compliance ranged from 0.5 to 1.75

Table 1: Distribution according to sex

\begin{tabular}{llll}
\hline Sl. no. & Sex & Numbers & Percentage \\
\hline 1 & Males & 15 & 30 \\
2 & Females & 35 & 70 \\
\hline Total & & 50 & 100 \\
\hline
\end{tabular}

Table 2: Distribution according to age group

\begin{tabular}{llll}
\hline Sl. no. & Age & Numbers & Percentage \\
\hline 1 & $18-25$ & 9 & 18 \\
2 & $26-35$ & 9 & 18 \\
3 & $36-45$ & 10 & 20 \\
4 & 45 and above & 22 & 44 \\
\hline Total & & 50 & 100 \\
\hline
\end{tabular}


(normal) in $86 \%$ of the cases (Table 5). The typmpanomeric curve was type A (normal) in $78 \%$ of the cases and type C in $8 \%$ of the cases (Table 6). At the end of 4 and 8 weeks,

Table 3: Distribution according to grade of retraction of tympanic membrane on examination

\begin{tabular}{llll}
\hline SI. no. & Grades & Numbers & Percentage \\
\hline 1 & Normal & 6 & 6 \\
2 & I & 57 & 57 \\
3 & II & 27 & 27 \\
4 & III & 8 & 8 \\
5 & IV & 2 & 2 \\
\hline Total & & 100 & 100 \\
\hline
\end{tabular}

Table 5: Distribution according to middle ear compliance

\begin{tabular}{llll}
\hline Sl. no. & Compliance & Numbers & Percentage \\
\hline 1 & $0.5-1.75$ (normal) & 86 & 86 \\
2 & $<0.5$ & 12 & 12 \\
3 & $>1.75$ & 2 & 2 \\
\hline Total & & 100 & 100 \\
\hline
\end{tabular}

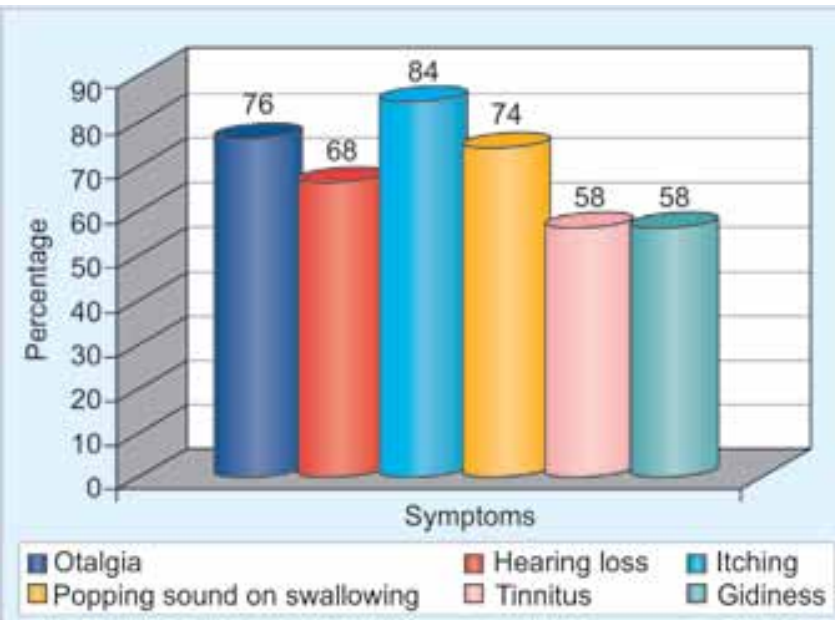

Graph 1: Distribution according to symptoms of Eustachian tube catarrh

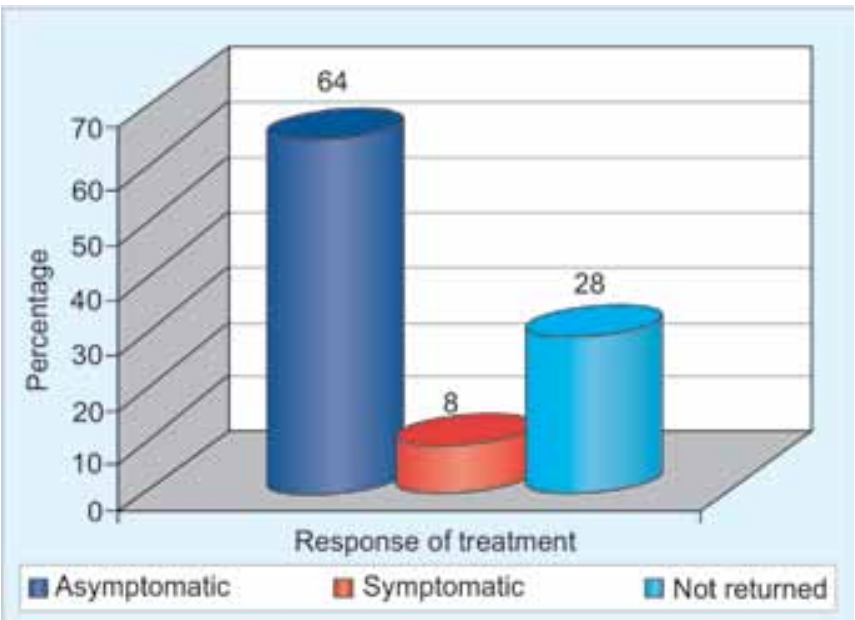

Graph 3: Distribution according to response of treatment at the end of 8 weeks the response of treatment to proton pump inhibitors was evaluated that became significant $(Z=3.53, p<0.05)$ in the studied group (Graphs 2 and 3).

Table 4: Distribution according to middle ear pressure

\begin{tabular}{llll}
\hline Sl. no. & Middle ear pressure & Numbers & Percentage \\
\hline 1 & 100 to +100 (normal) & 90 & 90 \\
2 & $\leq 100$ & 8 & 8 \\
3 & $\geq 100$ & 2 & 2 \\
\hline Total & & 100 & 100 \\
\hline
\end{tabular}

Table 6: Distribution according to tympanometric curve

\begin{tabular}{llll}
\hline Sl. no. & Compliance & Numbers & Percentage \\
\hline 1 & Type A (Normal) & 78 & 78 \\
2 & Type As & 12 & 12 \\
3 & Type C & 8 & 8 \\
4 & Type AD & 2 & 2 \\
\hline Total & & 100 & 100 \\
\hline
\end{tabular}

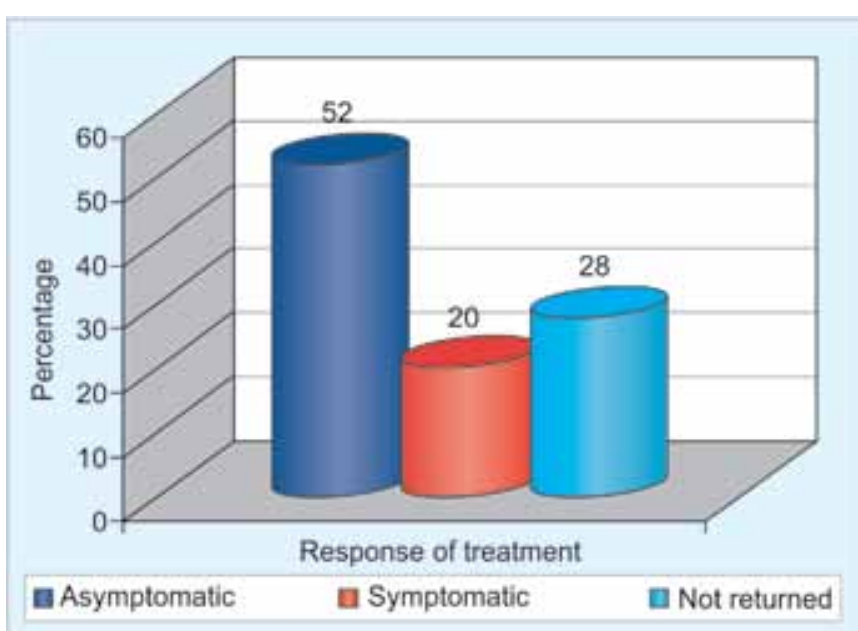

Graph 2: Distribution according to response of treatment at the end of 4 weeks

\section{DISCUSSION}

Eustachian tube is named after Bartolomeo Eustachio. It connects the tympanic cavity and the nasopharynx. Gastroesophageal reflux disease is defined as chronic symptoms due to mucosal damage caused by the reflux of gastric contents into the esophagus. The classical symptoms of GERD are heartburn, regurgitation, chest pain, dysphagia, odynophagia, nausea, dyspepsia, bloating, belching, indigestion, water brash and hiccups. ${ }^{1}$ The suspected or proven extraesophageal manifestations of GERD are depicted in Table $7 .^{2}$

Hawkins ${ }^{3}$ has termed LPR as a modern day 'great masquerader' and has stated that it is a new term given to gastroesophageal reflux leading to atypical symptoms in the upper aerodigestive tract. ${ }^{3}$ 
Table 7: Suspected or proven extraesophageal manifestations of GERD ${ }^{2}$

\begin{tabular}{ll}
\hline ENT & Pulmonary \\
\hline Pharyngitis & Asthma \\
Otitis & Cough \\
Sinusitis & Idiopathic pulmonary fibrosis \\
Vocal cord granulomas & Chronic bronchitis \\
Subglottic stenosis & Pneumonia \\
Laryngitis & \\
Hoarseness/voice changes & Miscellaneous \\
Globus & Chest pain \\
Laryngeal cancer & Sleep apnea \\
Cough & Dental erosions \\
\hline
\end{tabular}

The term LPR denotes gastroesophageal reflux that reaches above the esophageal sphincter. The mechanism of the otolaryngological manifestations of GERD is most likely the result of intermittent nocturnal gastroesopharyngeal reflux and contact of upper airway structures with refluxate. Upper esophageal sphincter pressures are lower at night, enhancing the opportunity of refluxate to cross this barrier and other protective or neutralizing mechanisms (cough, salivation) are also suppressed at night. Thus, many of the ENT manifestations of gastroesophageal reflux may depend to a large extent on the nocturnal reflux. ${ }^{2}$

Ulualp et $\mathrm{al}^{4}$ have done a study to determine the prevalence and the characteristics of pharyngeal acid reflux events in single and multiple otolaryngological disorders. The authors hypothesize that the presence of posterior laryngitis may be an indicator of a causal role for gastroesophageal reflux in other aerodigestive tract lesions, such as chronic rhinosinusitis, vocal cord nodule and laryngotracheal stenosis. ${ }^{4}$

Issing et $\mathrm{al}^{5}$ did a study in 40 patients to determine the incidence of gastroenterological disease in patients complaining of upper aerodigestive, pulmonary, laryngeal, pharyngeal and oral symptoms. They showed that in many patients suffering from the above-mentioned otolaryngological symptoms, occult gastroesophageal disease was present.

Koufman $^{6}$ did a clinical investigation of 225 patients to establish occult (silent) gastroesopshageal reflux. GERD is an etiological factor in the development of many inflammatory and neoplastic disorders of the upper aerodigestive tract. ${ }^{6}$

Catalano et $\mathrm{al}^{7}$ have done a study in a group of 110 patients with persistent upper respiratory symptoms and no suspicion of GERD symptoms to determine the prevalence of erosive and nonerosive esophagitis. They concluded that these patients had a higher prevalence of esophagitis as compared with group of similar age. They suggested that in patients with chronic upper respiratory symptoms, there is coexisting occult gastroesophageal reflux and the approach to each patient should be individualized. In patients with atypical symptoms or warning symptoms of dysphagia, odynophagia or weight loss, early diagnostic evaluation is suggested. Diagnostic evaluation should begin with endoscopy followed by ambulatory $\mathrm{pH}$ monitoring especially in patients with negative findings or persistent upper respiratory symptoms recalcitrant to therapy.

The therapeutic approaches to otolaryngological disorders associated with gastroesophageal reflux include lifestyle modifications, acid suppression therapy and surgical therapy.

Several studies report a decrease in distal esophageal acid exposure with the elevation of head-end of the bed, decreased fat intake, decreased smoking, avoiding recumbence for up to 3 hours postprandially. ${ }^{8}$

Steward et $\mathrm{al}^{9}$ have done a study to determine the efficacy of proton pump inhibitors for chronic laryngopharyngitis treated with lifestyle modification. Their study suggests that this significantly improves the symptoms attributable to the reflux, with or without proton pump inhibitor therapy. Thus, they suggest lifestyle modification for 2 months, a reasonable alternative to medical therapy for chronic laryngopharyngitis secondary to acid reflux. ${ }^{9}$ Empirical trials with proton pump inhibitors have revealed 8 weeks as the optimal duration for treatment in patients with otolaryngological symptoms, such as posterior laryngitis. ${ }^{8}$

Politzer hypothesized that ETD was an important factor in the pathogenesis of middle ear disease in the 1860s. Much research has been conducted to better understand the role of ETD in otitis media with effusion.

Eustachian tube obstruction can result in altered middle ear function. The obstruction can be mechanical, functional or both. Acute salpingitis is the first stage in development of acute supporative otitis media. At times, the respiratory epithelium lining of the tube may become congested with inflammatory swelling or produce an excessive secretion, resulting in blockage of the tube and some deafness.

There is increasing evidence that GERD causes laryngeal signs and symptoms. This is often referred to as reflux laryngitis or LPR. In fact, it is estimated that 4 to $10 \%$ of patients presenting to the ENT. Nose and throat physician do so because of symptoms that are, in part, related to GERD. ${ }^{10}$

White et $\mathrm{al}^{11}$ have done a study to explore the possible relationship between gastroesophageal reflux and ETD in an animal model. They concluded that nasopharyngeal exposure to stimulate gastric juice causes ETD in rats; specifically, middle ear pressure regulation and mucociliary clearance of middle ear contents were 
disabled. ${ }^{11}$ This supports the linking of nasopharyngeal reflux to ETD and secondary development of otitis media. Sudhoff et $\mathrm{al}^{12}$ have done a study to trace gastric reflux and to examine whether it reaches middle ear in a Mongolian gerbil model. They concluded that in animals with traceable laryngeal reflux the ink (they had injected Chinese ink into the stomach to trace the path of a potential gastroesophageal reflux) advanced through Eustachian tube and reached the middle ear. They also found that when reflux reaches middle ear on one side it also reaches the contralateral middle ear in most cases. Heavner et $\mathrm{al}^{13}$ have done a study to delineate the relationship between gastroesophageal reflux and Eustachian tube function. This study was done on rats and it suggested that middle ear exposure to pepsin/ hydrochloric acid $(\mathrm{HCl})$ leads to ETD in rats. ${ }^{13}$

In our study of 50 adult patients with Eustachian tube catarrh, itching in the ears was the commonest symptom $(84 \%)$. Other symptoms were otalgia $(76 \%)$, popping sensation on swallowing $(74 \%)$, hearing loss $(66 \%)$, tinnitus (56\%) and giddiness (56\%). The symptoms of ETD are fullness, pain in the ears, hearing loss, ringing in the ears and dizziness. Itching was not reported as a symptom of Eustachian tube catarrh in other studies. ${ }^{14}$ The possible cause of itching is the mild stretching and retraction of the tympanic membrane due to negative pressure in the middle ear following prolonged Eustachian tube block.

The group consisted of $15(30 \%)$ men and $35(70 \%)$ women with a mean age group of $36.98+11.67$ (mean + standard deviation). The majority of the patients were of 45 years and above. Gastroesophageal reflux is more common in an older age group. It has been documented that there is an increased incidence in reflux and esophageal contact time in adults older than 50 years. This is likely due to combination of increased incidence of hiatus hernia, diminished peristaltic amplitudes and reduced salivary response to esophageal acid contact. ${ }^{15}$

The most common presenting symptoms of GERD are heartburn and regurgitation. This pattern is often referred as typical GERD. Patients with extraesophageal manifestations often do not complain of the classical symptoms. ${ }^{16}$

In our study of patients with Eustachian tube catarrh (without local cause, e.g. upper respiratory tract infection, allergy), $78 \%$ had to or more typical symptoms of GERD. The remaining (22\%) did not have any of the typical symptoms.

Hoarseness is caused by GERD in $10 \%$ of cases. Chronic laryngitis and persistent cough are associated with reflux in $60 \%$ of patients. Gastroesophageal reflux disease is the third leading cause of chronic cough in $20 \%$ of the cases. Globus sensation is caused by GERD in 25 to $50 \%$ of the cases. ${ }^{17}$
In our study, $32 \%$ of patients had two or more symptoms of laryngitis. On indirect laryngoscopy examination, they also had congestion of the arytenoids.

A negative middle ear pressure less than $(-100)$ curve was found in $8 \%$ of studied cases. Type C curve was found in $8 \%>$ of the cases and tympanic membrane retraction on otoscopic examination more than grade II was found in $10 \%$ of the cases. A normal tympanic membrane was seen in $6 \%$, grade I retraction in $57 \%$ and grade II retraction in $27 \%$ cases. Our study showed that all patients with symptoms of Eustachian tube catarrh had a negative Eustachian tube function test (Williams' test). Major changes in the pressure of the middle ear need not appear in Eustachian tube catarrh; they may be time dependent.

In our study, all the patients of Eustachian tube catarrh with and without symptoms of gastroesophageal reflux were treated with proton pump inhibitors. Patients were followed up at an interval of 4 weeks to a maximum of 3 months. Our study shows that at the end of 4 weeks $52 \%$ of the patients had improvement, $20 \%$ had no improvement and $28 \%$ did not return for follow-up. Response of treatment to proton pump inhibitors was significant $(Z=3.53, p<0.05)$ at the end of 4 weeks.

All patients who did not respond to treatment at the end of 4 weeks were continued on the same treatment for another 4 weeks at the end of which $60 \%$ responded, $40 \%$ did not respond to the treatment. This group of four patients who did not respond to treatment was again given treatment for another 4 weeks, at the end of which they were all asymptomatic.

At the end of 8 weeks, $64 \%$ patients had responded, $8 \%$ were symptomatic and $28 \%$ had not returned for follow-up.

Bilgen et $\mathrm{al}^{18}$ studied 36 patients with symptoms suggestive of LPR. In these patients, they compared proton pump inhibitor therapy as a method of diagnosis of LPR with 24 hours double probe $\mathrm{pH}$ monitoring.

They concluded that empirical trial of proton pump inhibitors provides reliable information about the relationship between the pharyngeal reflux and the laryngopharyngeal symptoms. The improvement in symptoms in 2 months confirms the presence of this relationship.

In our study also, response to treatment to proton pump inhibitors was highly significant $(Z=7.14, \mathrm{p}<$ $0.001)$ at the end of 8 weeks. This supports the hypothesis that Eustachian tube catarrh could be due to LPR. It also suggests that proton pump inhibitors are effective in the treatment of Eustachian tube catarrh (without features of local disease) in higher doses and with regular follow-up.

Further studies on larger population will be required to establish a comparative effect of different proton pump inhibitors on Eustachian tube catarrh. 


\section{ACKNOWLEDGMENT}

The authors are greatly indebted to all members of the Department of ENT and Head and Neck Surgery, Department of Biostatistics and Department of Electronic Data Processing (EDP), Pondicherry Institute of Medical Sciences, Puducherry for their cooperation.

\section{REFERENCES}

1. Younes Z, Johnson DA. Diagnostic evaluation in gastroesophageal reflux disease. Gastroenterol Clin North Am 1999 Dec;28(4):809-830.

2. Fennerty MB. Extraesophageal gastroesophageal reflux disease: presentations and approach to treatment. Gastroenterol Clin North Am 1999 Dec;28(4):861-873.

3. Hawkins BL. Laryngopharyngeal reflex: a modern day 'great masquerader'. J Ky Med Assoc 1997 Sep;95(9):379-385.

4. Ulualp SO, Toohill RJ, Shaker R. Pharyngeal acid reflux in patients with single and multiple otolaryngologic disorders. Otolaryngol Head Neck Surg 1999 Dec;121(6):725-730.

5. Issing WJ, Gross M, Tauber S. Manifestations of gastroesophageal reflux in the otorhinolaryngology tract. Laryngorhinootologie 2001 Aug;80(8):464-469.

6. Koufman JA. The otolaryngologic manifestations of gastroesophageal reflux disease: a clinical investigation of 225 patients using ambulatory 24-hour $\mathrm{pH}$ monitoring and an experimental investigation of the role of acid and pepsin in the development of laryngeal injury. Laryngoscope 1991 Apr;101(4pt 2 suppl 53):1-78.

7. Catalano F, Terminella C, Grillo C, Biondi S, Zappala M, Bentivegna C. Prevalence of oesophagitis in patients with persistent upper respiratory symptoms. J Laryngol Otol 2004 Nov;118(11):857-861.

8. Ulualp SO, Toohill RJ. Laryngopharyngeal reflux. Otolaryngol Clin North Am 2000 Aug;33(4):789-790.
9. Steward DL, Wilson KM, Kelly DH, Patil MS, Schwartzbauer HR, Long JD, Welge JA. Proton pump inhibitor therapy for chronic laryngopharyngitis: a randomized placebo-control trial. Otolaryngol Head Neck Surg 2004 Oct;131(4):342-350.

10. Cummings CW, Flint PW, Harker LA, Haughley BH, Richardson MA, Robbins KT, et al. Cummings otolaryngology head and neck surgery. 4th ed. Vol. 2. Philadelphia, Pennsylvania: Elsevier Mosby; 2005. p. 1855-1860.

11. White DR, Heavner SB, Hardy SM, Prazma J. Gastroesophageal reflux and Eustachian tube dysfunction in an animal model. Laryngoscope 2002 Jun;112(6):955-961.

12. Sudhoff H, Bucker R, Groll C, Shagdarsuren S, Dazert S, Schreiber S. Tracing of gastric reflux into the middle ear in a Mongolian Gerbil Model. Otol Neurotol 2007 Jan;28(1): 124-128.

13. Heavner SB, Hardy SM, White DR, McQueen CT, Prazma J, Pillsbury HC 3rd. Function of the Eustachian tube after weekly exposure to pepsin/hydrochloric acid. Otolaryngol Head Neck Surg 2001 Sep;125(3):123-129.

14. Hoffmann D. Eustachian tube dysfunction-1. Available at: http://www.doctor Hoffmann/etd.htm. Accessed November 23, 2007.

15. Orr WC, Chen CL. Aging and neural control of the GI tract: IV. Clinical and physiological aspects of gastrointestinal motility and aging. Am J Physiol Gastrointest Liver Physiol 2002 Dec;283(6):G1226-G1231.

16. Vaezi MF. Extraoesophageal manifestations of gastroesophageal reflux: a focus on laryngeal manifestations of gastroesophageal reflux disease-4. Available at: www.gastro.org. June 6, 2007.

17. Vaezi MF. Extraesophageal manifestations of gastroesophageal reflux disease. Clin Cornerstone 2003;5(4):32-38.

18. Bilgen C, Ogut F, Kesimli-Dinc H, Kirazli T, Bor S. The comparison of an empiric proton pump inhibitor trial vs 24 hours double probe $\mathrm{pH}$ monitoring in laryngopharyngeal reflux. J Laryngol Otol 2003 May;117(5):386-390. 